\title{
16-Channel Low Power CMOS Integrated Circuit for Detection of Auditory Nerve Signals
}

\author{
Jimin Cheon \\ School of Electronic Engineering, Kumoh National Institute of Technology, \\ Gumi, Korea \\ jimin.cheon@kumoh.ac.kr
}

\begin{abstract}
For sensorineural hearing loss, auditory perception can be activated by electrical stimulation of a nervous system via electrode implanted into cochlea or auditory nerves. As the tonotopic map of the human auditory nerve has not been absolutely identified, the recording of its signal with electrode is desirable to determine the map. This paper proposes a 16-channel analog front-end for auditory nerve signal detection. Its channel consists of an $A C$ coupling circuit, a 4th-order Gm-C lowpass filter (LPF), and a single-slope analog-todigital converter (ADC). While blocking DC level, the AC coupling circuit transfers only AC signal. The Gm-C LPF is designed with operational transconductance amplifiers (OTAs) using floating-gate technique for small Gm. The channel-wise single-slope ADC is used because it has the small area and low power consumption. Experiment results show the prototype chip fabricated in 0.35- $\mu \mathrm{m}$ CMOS process has the bandwidth of $0.1 \sim 6.95 \mathrm{kHz}$ with the gain of $19.9 \mathrm{~dB}$, the effective resolution of 7.7 bits and the power consumption per a channel of $12 \mu \mathrm{W}$.
\end{abstract}

Keywords: analog front-end, auditory nerve, electrode, floating-gate, Gm-C filter

\section{Introduction}

Hearing impairments are divided into conductive hearing loss and sensorineural hearing loss. The solutions to sensorineural hearing loss become more important, whereas conductive hearing loss can be corrected by a surgical operation. Among many solutions, one way is to use the auditory prosthesis which can recover the hearing ability by substituting electrical circuits with electrodes for malfunctioned auditory pathways $[1,2]$. However, since the tonotopic map of the human auditory nerve has not been absolutely identified, the recording of the auditory nerve signal with electrode is desirable to determine the tonotopic map [3-7].

This paper proposes the low power 16-channel analog front-end for auditory nerve signal recording. A channel of the proposed analog front-end consists of an AC coupling circuit, a low-power 4th-order Gm-C lowpass filter (LPF), and a single-slope analog-to-digital converter (ADC). The LPF is implemented with operational transconductance amplifiers (OTAs) using floating gate technique [8] and a gain amplifier with the gain of 10 is embedded in the LPF to reduce the power consumption. Also, the readout speed is improved by adopting channel-parallel ADC structure, when compared with a conventional single ADC structure.

The rest of the paper is organized as follows. Next, the architecture of the proposed analog front- end is described. Section 3 provides the detailed circuit design. Section 4 
shows the experimental results of the fabricated chip. Finally, conclusions are provided in Section 5 .

\section{Structure of the Proposed Analog Front-End}

Figure 1 shows the architecture of the proposed analog front-end to detect auditory nerve signal. The analog front-end detects nerve signal from auditory nerve by using an array of 16-channel electrodes. The AC coupling circuit, which consists of a capacitor and a PMOS transistor, transfers nerve signal without the effect of the uncertain DC voltage level. The proposed Gm-C LPF is designed with the cutoff frequency of $7 \mathrm{kHz}$ and the gain amplifier with the gain of $20 \mathrm{~dB}$ is embedded in the LPF to reduce power consumption, when compared with the case that the amplifier is designed separately. After 16-channel nerve signals are filtered, they are simultaneously digitized by the 16channel ADCs. A channel-parallel ADC is designed as the structure of a single-slope ADC which occupies the small silicon area, because the ADC needs the sampling rate of $40 \mathrm{kS} / \mathrm{s}$ and the resolution of 8 bit to detect auditory nerve signal. Also, the digitized data are readout by a serializer to reduce the number of the output interface lines.

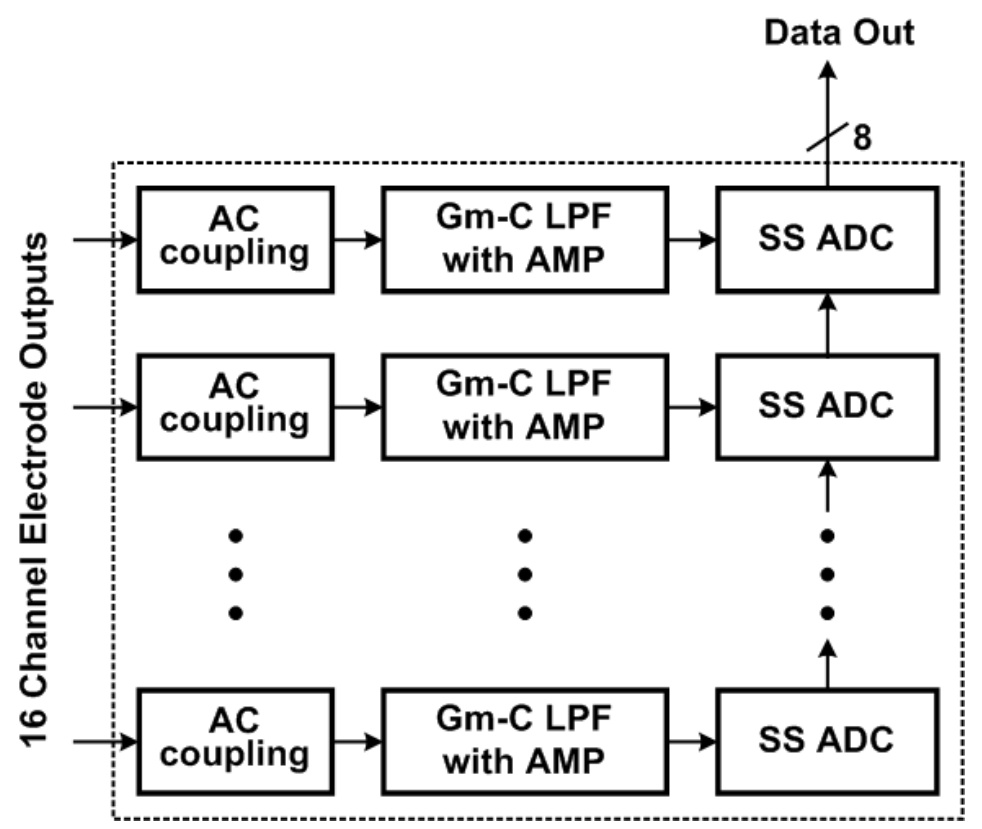

Figure 1. Block Diagram of the Proposed 16-channel Analog front-end for Auditory Nerve Signal Detection

\section{Circuit Implementation of the Proposed Analog Front-End}

\subsection{AC Coupling Circuit}

Figure 2 shows the AC coupling circuit that transfers only AC signal, while blocking DC level. Since auditory nerve signal detected by the electrode is in a floating state, the AC coupling circuit is needed to provide the proper input common-mode voltage for the operation of the LPF. Although the conventional AC coupling circuit is implemented with the combination of the external large capacitor and resistor, the proposed AC 
coupling circuit is implemented with the small capacitor $\mathrm{C}_{\mathrm{ac}}$ and the large channel resistance of the transistor $\mathrm{M}_{\mathrm{ac}}$ controlled by the bias voltage $\mathrm{V}_{\mathrm{b}}$, because the proposed analog front-end is an integrated chip (IC) and its silicon area is reduced.

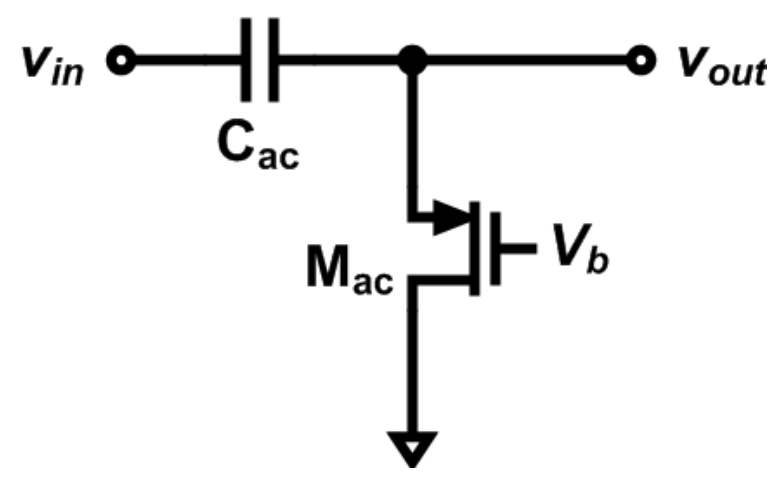

Figure 2. AC Coupling Circuit

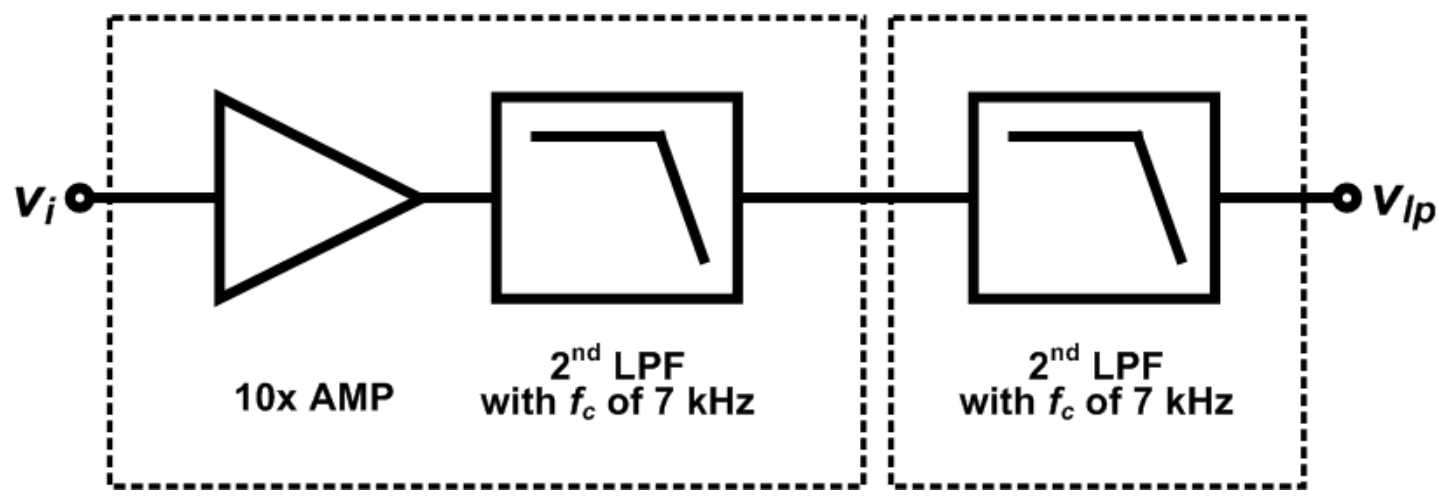

Figure 3. Block Diagram of the Proposed 4th-order Gm-C LPF with a 10x Amplifier

\subsection{Gm-C LPF}

Figure 3 shows the block diagram of the proposed 4th-order LPF. The proposed 4thorder LPF has the cascade structure that successively consists of two 2nd-order Gm-C LPFs. Also, the 2nd-order LPF at the first stage embeds the amplifier with the gain of $20 \mathrm{~dB}$ to amplify auditory nerve signal which has the small signal range and reduce power consumption and silicon area, when compared with the case that the amplifier is designed separately.

Figure 4 shows the schematic diagram and the signal flow graph of the 2nd-order Gm-C LPF that is the functional block of the proposed 4th-order Gm-C LPF. The 2ndorder $\mathrm{Gm}-\mathrm{C}$ LPF is implemented with three OTAs with three transconductances $\mathrm{g}_{\mathrm{m} 1}$, $\mathrm{g}_{\mathrm{m} 2}$, and $\mathrm{g}_{\mathrm{m} 3}$. If $\mathrm{g}_{\mathrm{m} 2}=\mathrm{g}_{\mathrm{m} 3}=\mathrm{g}_{\mathrm{m}}$ and $\mathrm{C}_{1}=\mathrm{C}_{2}=\mathrm{C}$, the transfer function of the 2nd-order LPF can be obtained as Equation (1) from the signal flow graph.

$$
\frac{V_{L P}(s)}{V_{I}(s)}=\frac{\frac{g_{m 1} g_{m}}{C^{2}}}{s^{2}+\frac{2 g_{m}}{C} s+\frac{g_{m}^{2}}{C^{2}}}
$$


Here, the voltage gain of the 2nd-order LPF, $A_{v}$ is obtained as $g_{m 1} / g_{m} V / V$ and the cutoff frequency $\mathrm{f}_{\mathrm{c}}$ is obtained as $\mathrm{g}_{\mathrm{m}} / 2 \pi \mathrm{C} \mathrm{Hz}$.

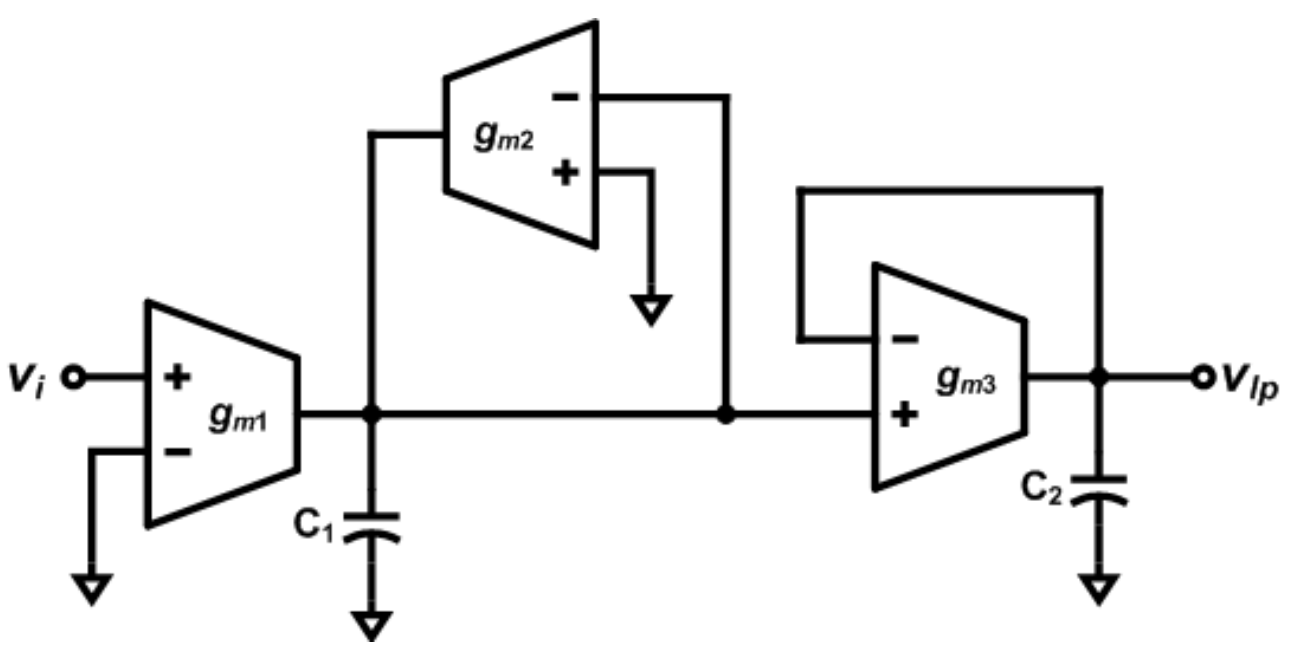

$(\alpha)$

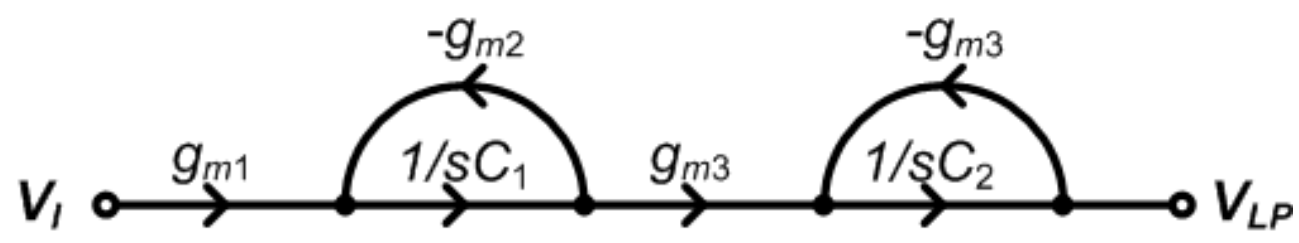

( $\beta)$

Figure 4. (a) Schematic Diagram and (b) Signal Flow Graph of the 2nd-order Gm-C LPF

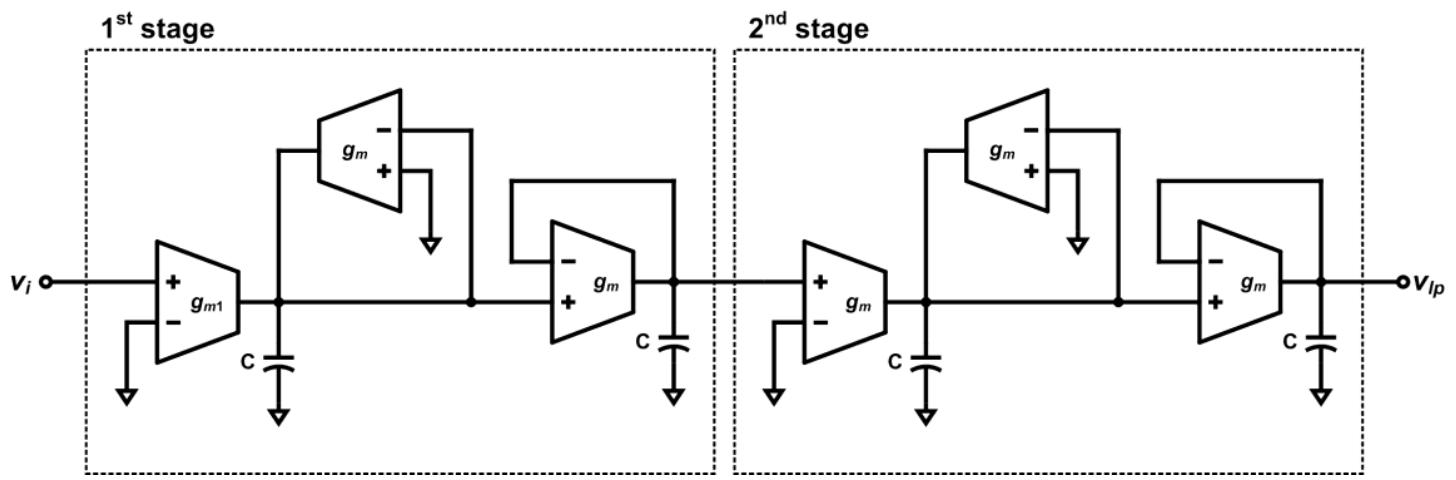

Figure 5. Schematic Diagram of the 4th-order Gm-C LPF

Figure 5 shows the total schematic diagram of the 4th-order Gm-C LPF used in this paper. The 4th-order Gm-C LPF is implemented as the cascade structure that successively consists of two 2nd-order Gm-C LPFs and has the voltage gain of $20 \mathrm{~dB}$ that is adjusted by the ratio of $\mathrm{g}_{\mathrm{m} 1}$ and $\mathrm{g}_{\mathrm{m}}$ in the 2nd-order LPF at the first stage. The 2nd-order LPF at the second stage has the voltage gain of $0 \mathrm{~dB}$, because all OTAs have the same transconductance. Table 1 shows 
the parameters of the 4th-order Gm-C LPF to achieve the voltage gain of $20 \mathrm{~dB}$ and the cutoff frequency of $7 \mathrm{kHz}$.

Table 1. Parameters of the 4th-order Gm-C LPF

\begin{tabular}{c|c||c}
\hline \hline \multirow{2}{*}{ Transconductance } & $g_{m 1}$ & $682 \mathrm{nA} / \mathrm{V}$ \\
\cline { 2 - 3 } & $g_{m}$ & $68.1 \mathrm{nA} / \mathrm{V}$ \\
\hline Capacitance & $\mathrm{C}$ & $1.55 \mathrm{pF}$ \\
\hline \hline
\end{tabular}

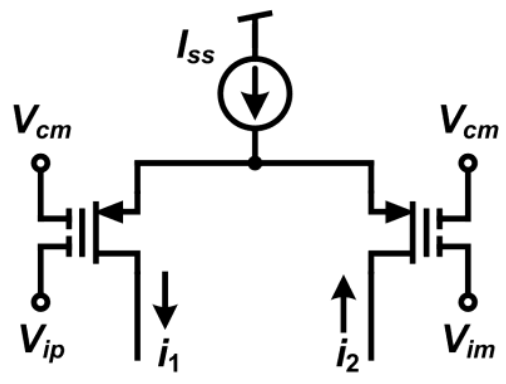

$(\alpha)$

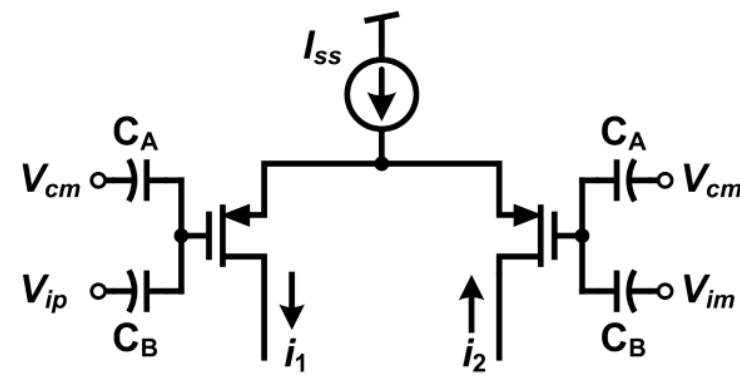

( $\beta)$

Figure 6. Transconductance Reduction Technique with Floating Gate

Figure 6 shows the transconductance reduction technique with floating gate. The floating gate technique is used in the input stage of the conventional OTA to achieve smaller transconductance than that of the conventional OTA. Instead of using double poly CMOS process as shown in Figure 6 (a), the same result can be obtained by using two capacitors implemented from the normal CMOS process as shown in Figure 6 (b). The effective transconductance, $\mathrm{G}_{\mathrm{m}}$ of the proposed OTA is decreased as Equation (2) by the floating gate technique.

$$
G_{m}=\frac{C_{A}}{C_{A}+C_{B}} g_{m}
$$

where $g_{m}$ is the transconductance of the input transistor. Figure 7 shows the schematic of the OTA using floating gate technique. 


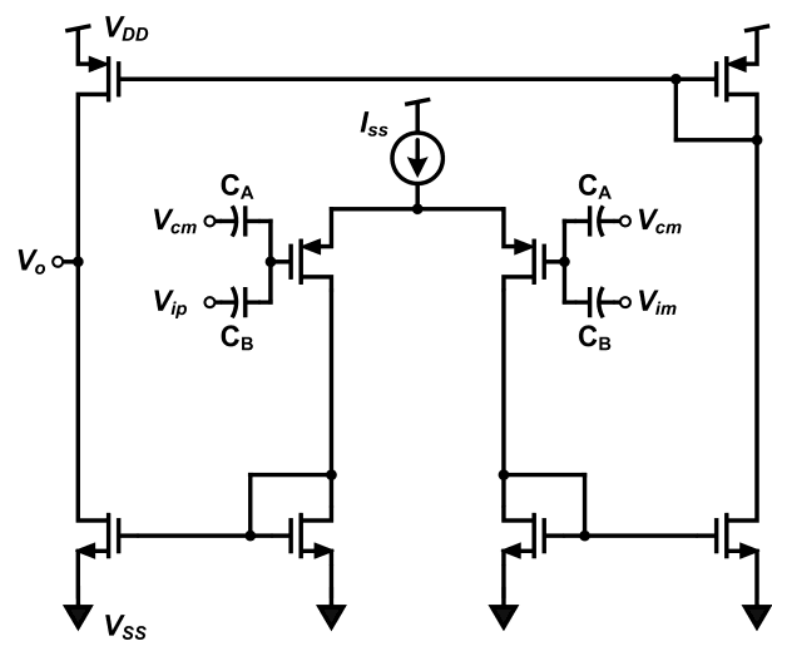

Figure 7. Schematic of the OTA using Floating Gate Technique

\subsection{Single-Slope ADC}

Figure 8 shows the block diagram of the channel-parallel single-slope ADC. The output of the LPF is sampled/held by the switch and the capacitor $\mathrm{C}_{\text {sh }}$. Then the 8-bit counter starts and the ramp signal ramps concurrently. When the ramp signal exceeds the voltage sampled in $\mathrm{C}_{\mathrm{sh}}$, the output of the comparator changes from 'Low' state to 'High' state. At this moment, the register latches the counter value [9]. This counter value is the digitized data of the LPF output.

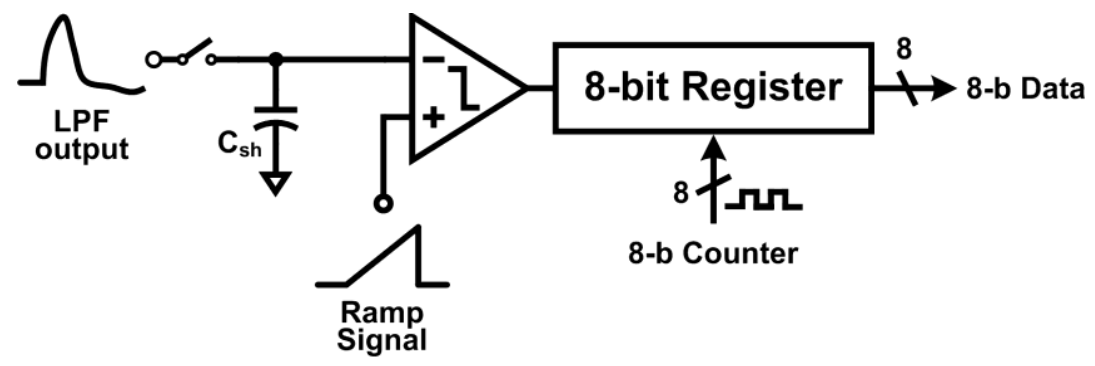

Figure 8. Block Diagram of the channel-parallel single-slope ADC

\section{Experimental Results}

The layout of the fabricated chip is shown in Figure 9. The chip is fabricated with $0.35-\mu \mathrm{m} 1$-poly 4-metal CMOS process and the core area is $2.6 \mathrm{~mm} \times 3.7 \mathrm{~mm}$ and operates at power supply of $3.0 \mathrm{~V}$. The AC coupling circuit and the Gm-C LPF have the input common-mode voltage of $1.5 \mathrm{~V}$. The input ranges of the Gm-C LPF and the single-slope ADC are $100 \mathrm{mV}$ and $1 \mathrm{~V}$ respectively. The ADC has the sampling rate of $40 \mathrm{kS} / \mathrm{s}$.

Figure 10 shows the photograph of the test PCB board for the prototype chip. The power supply, current, reference voltage, and control signal are provided into the inside of the chip from mother board through the flexible cable. Also, the digitized output can be transferred through the flexible cable. 

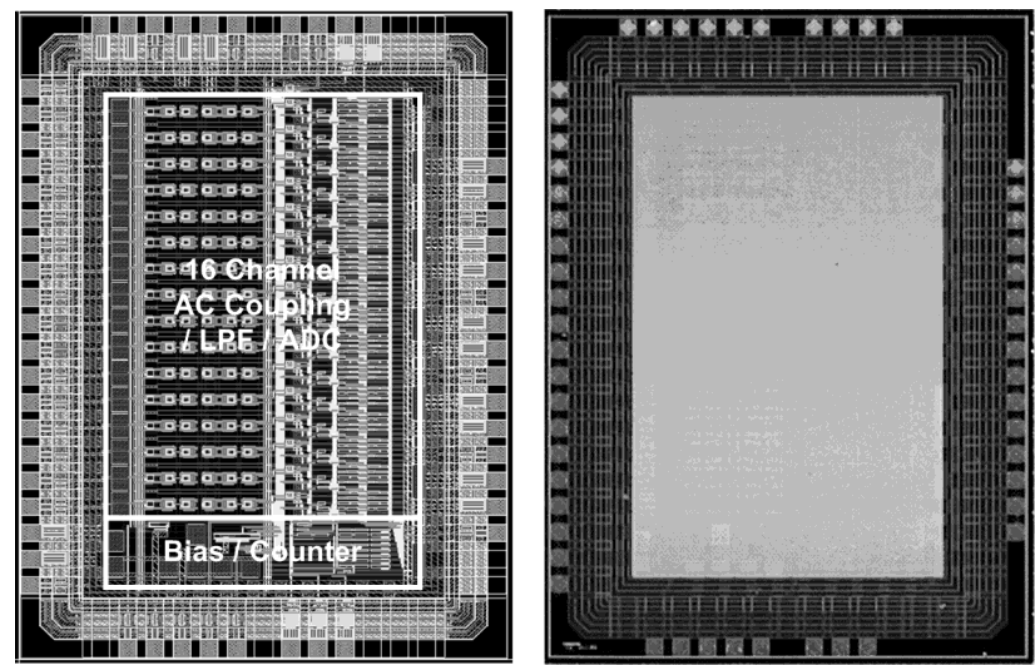

Figure 9. Layout and Microphotograph of the Prototype Chip for the Proposed Analog Front-End

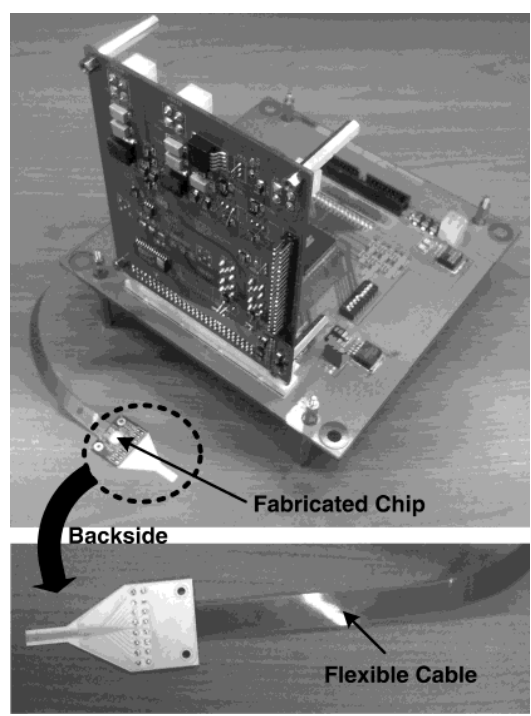

Figure 10. Photograph of the Test Board for the Prototype Chip

Figure 11 shows the comparison between the simulation result and the experimental result of the frequency response for the sinusoidal input signal with the magnitude of 80 $\mathrm{mV}$. It is observed that the signal under the frequency of $100 \mathrm{~Hz}$ is attenuated, the cutoff frequency is about $6.95 \mathrm{kHz}$, and the passband gain is about $19.9 \mathrm{~dB}$ as expected by the simulation.

Figure 12 shows the measured output spectrum of the single-slope ADC for $-8 \mathrm{~dB}_{\mathrm{FS}}, 1$ $\mathrm{kHz}$ sinusoidal input. The measured signal to noise-plus-distortion ratio (SNDR) and effective resolution are $48 \mathrm{~dB}$ and 7.7 bits respectively.

The power consumption per a channel is measured as $12 \mu \mathrm{W}$. Table 2 shows the performance summary. 


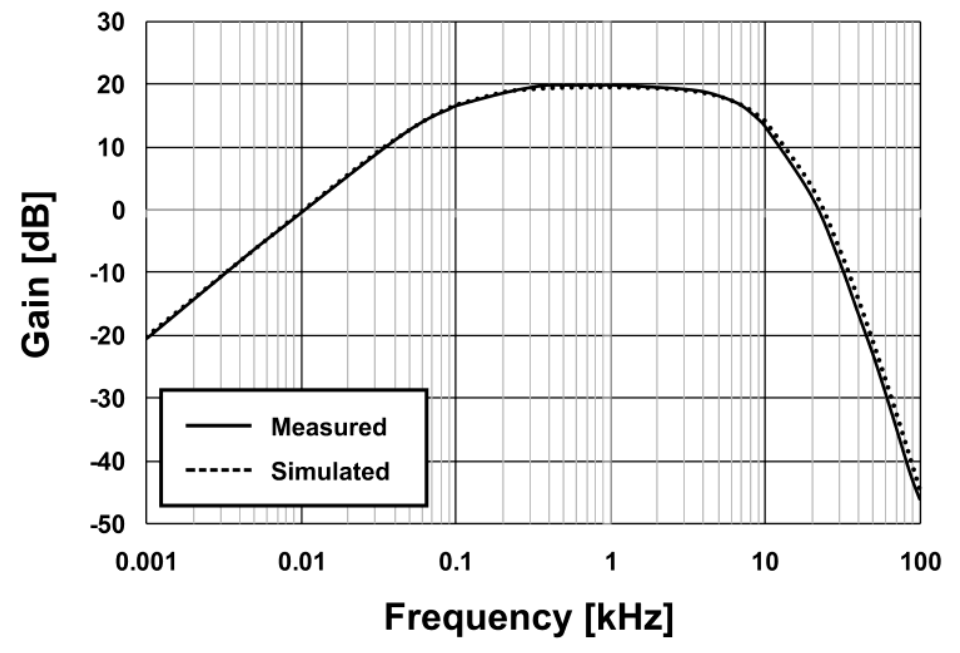

Figure 11. Frequency Response for the Sinusoidal Input Signal with the Magnitude of $80 \mathrm{mV}$

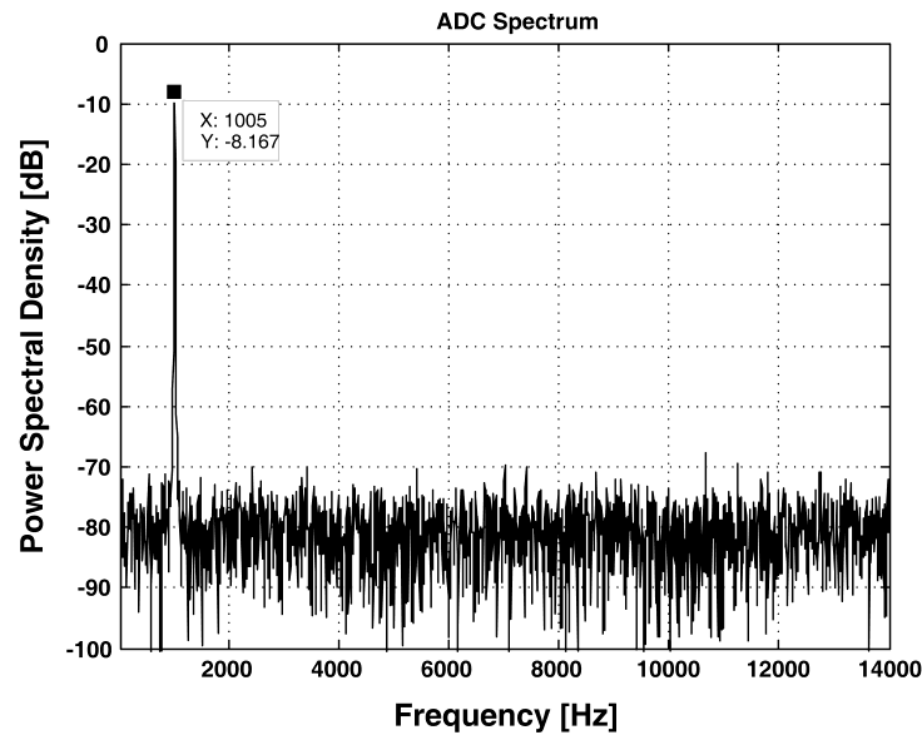

Figure 12. Measured Output Spectrum of $A D C$ for $-8 \mathrm{~dB}_{\mathrm{FS}}, 1 \mathrm{kHz}$ sinusoidal input

Table 2. Performance Summary

\begin{tabular}{c||c}
\hline \hline Technology & $0.35-\mu \mathrm{m} \mathrm{1P} 4 \mathrm{M}$ \\
\hline Supply voltage & $3.0 \mathrm{~V}$ \\
\hline Gm-C LPF cutoff frequency & $6.95 \mathrm{kHz}$ \\
\hline
\end{tabular}




\begin{tabular}{c||c}
\hline Gm-C LPF gain & $19.9 \mathrm{~dB}$ \\
\hline ADC sampling rate & $40 \mathrm{kS} / \mathrm{s}$ \\
\hline Effective resolution & $7.7 \mathrm{bits}$ \\
\hline ADC INL/DNL & $< \pm 0.5 \mathrm{LSB}$ \\
\hline $\begin{array}{c}\text { Power consumption } \\
\text { per a channel }\end{array}$ & $12 \mu \mathrm{W}$ \\
\hline \hline
\end{tabular}

\section{Conclusions}

We proposed the 16-channel analog front-end for auditory nerve signal detection. A channel of the proposed analog front-end consists of an AC coupling circuit, a low-power 4th-order Gm-C LPF, and a single-slope ADC. The AC coupling circuit transfers only AC signal while it blocks DC signal level. Considering the bandwidth of the auditory signal, the Gm-C LPF is designed with OTAs adopting floating-gate technique. For the channel-parallel ADC structure, the single-slope ADC is used because it occupies the small silicon area. Experimental results shows that the AC coupling circuit and LPF have the bandwidth of 100 $\mathrm{Hz} \sim 6.95 \mathrm{kHz}$ and the ADC has the effective resolution of 7.7 bits. The power consumption per a channel is $12 \mu \mathrm{W}$, the power supply is $3.0 \mathrm{~V}$, and the core area is $2.6 \mathrm{~mm} \times 3.7 \mathrm{~mm}$. The proposed analog front-end was fabricated in a 1-poly 4-metal $0.35-\mu \mathrm{m}$ CMOS process.

\section{Acknowledgements}

This paper was supported by Research Fund, Kumoh National Institute of Technology.

\section{References}

[1] F. -G. Zeng, A. Popper, and R. Fay, "Cochlear implants: electric hearing and auditory prostheses", NY: Springer-Verlag, New York (2004).

[2] D. J. Anderson, "Penetrating multichannel stimulation and recording electrodes in auditory prosthesis research," Hearing Research, vol. 242, no. 1-2, (2008), pp. 31-41.

[3] J. Wu, W. -F. Feng, and W. C. Tang, "A multi-channel low-power circuit for implantable auditory neural recording microsystems," International Conference of Biomedical Engineering, Paper \#4B1-05, CD-ROM, (2005).

[4] R. R. Harrison, P. T. Watkins, R. J. Kier, R. O. Lovejoy, D. J. Black, B. Greger, and F. Solzbacher, “A LowPower Integrated Circuit for a Wireless 100-Electrode Neural Recording System," IEEE Journal of SolidState Circuits, vol. 42, no. 1, (2007), pp. 123-133.

[5] D. Oertel, R. Bal, S. M. Gardner, P. H. Smith, and P. X. Joris, "Detection of synchrony in the activity of auditory nerve fibers by octopus cells of the mammalian cochlear nucleus," Proceedings of the National Academy of Sciences of the United States of America, vol. 97, no. 22, (2004), pp. 11773-11779.

[6] K. Najafi and K. D. Wise, "An implantable multielectrode array with on-chip signal processing," IEEE Journal of Solid-State Circuits, vol. 21, no. 6, (1986), pp. 1035-1044.

[7] T. Jochum, T. Denison, and P. Wolf, "Integrated circuit amplifiers for multi-electrode intracortical recording," Journal of Neural Engineering, vol. 6, no. 1, (2009), pp. 1-26.

[8] P. Garde, "Transconductance cancellation for operational amplifiers," IEEE Journal of Solid-State Circuits, vol. SC-12, (1977), pp. 310-311.

[9] N. Marston, "Solid-state imaging: a critique of the CMOS sensor", Ph.D. dissertation, Univ. of Edinburgh, Edinburgh, United Kingdom, (1998). 


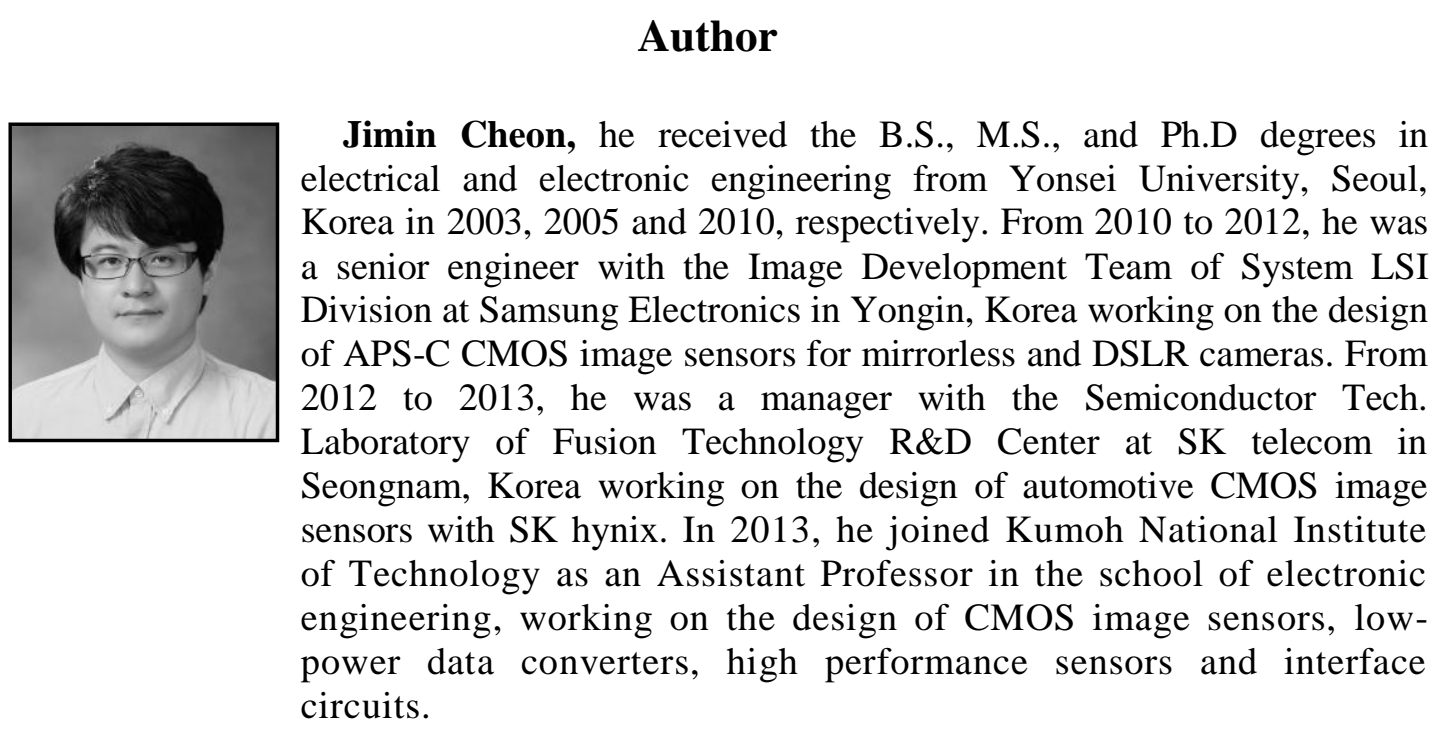

